Supporting Information

\title{
Facile Preparation of Continuous and Porous Polyimide Aerogel Fibers for Multifunctional Applications
}

\author{
Mengmeng Li, ${ }^{\dagger}$ Feng Gan, ${ }^{\ddagger}$ Jie Dong, ${ }^{*}, \dagger$ Yuting Fang, ${ }^{\dagger}$ Xin Zhao, ${ }^{\dagger}$ Qinghua Zhang ${ }^{*}, \dagger$
}

†State Key Laboratory for Modification of Chemical Fibers and Polymer Materials, College of Materials Science and Engineering, Donghua University, Shanghai 201620, PR China

‡School of Textile Materials and Engineering, Wuyi University, Jiangmen, Guangdong 529020, PR China

*Corresponding author: dj01@dhu.edu.cn; qhzhang@dhu.edu.cn

Preparation of the PAF fabric. A simple artificial manual weaving device (as shown in the following figure, length $(40 \mathrm{~cm}) \times$ wide $(20 \mathrm{~cm})$, distance between the adjacent tow warps is 5 $\mathrm{mm}$ ) was applied in fabricating the PAF textile. The obtained PAF-5 textiles have about three warps and eight wefts in per square centimeter. Areal density of the PAF-5 textile is approximately (28-35) $\mathrm{g} / \mathrm{m}^{2}$.

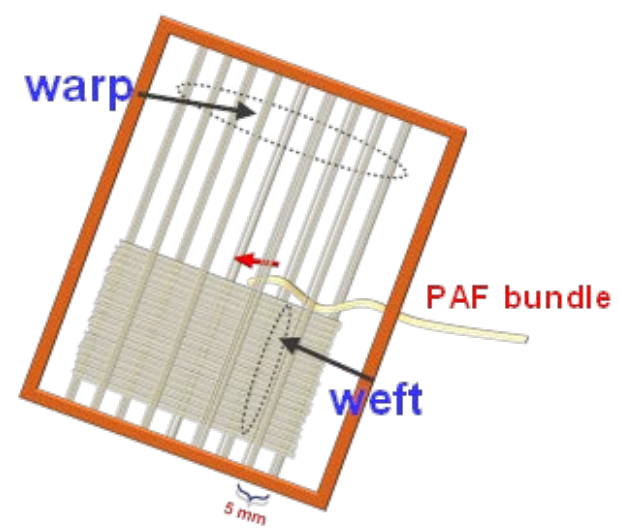

Figure S1. The schematic of a simple artificial manual weaving device for preparing PAF textiles 


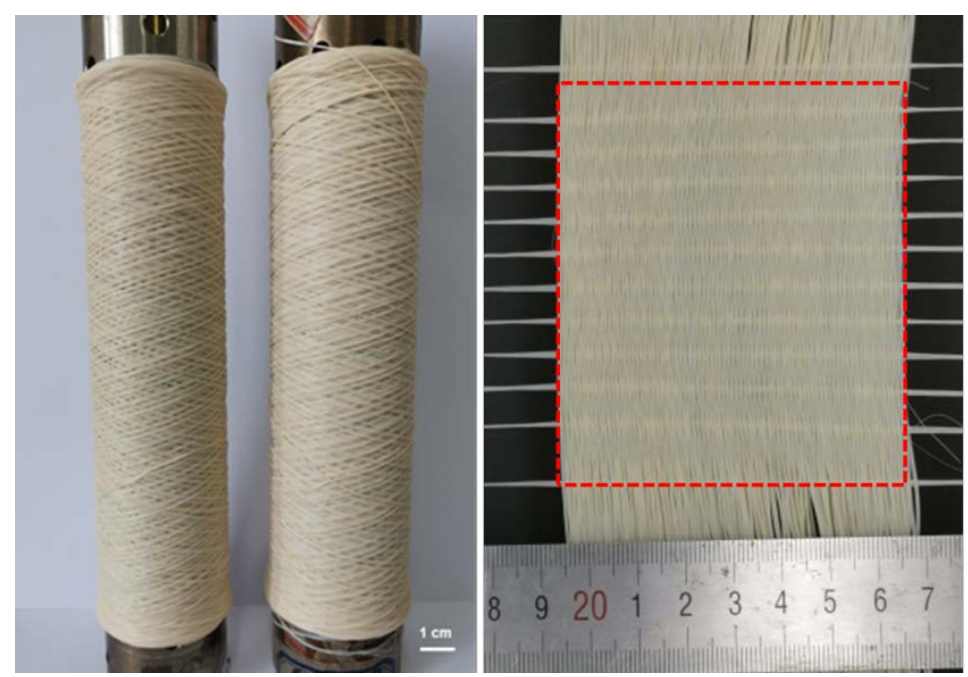

Figure S2. Optical image of the prepared PAF and a woven textile.
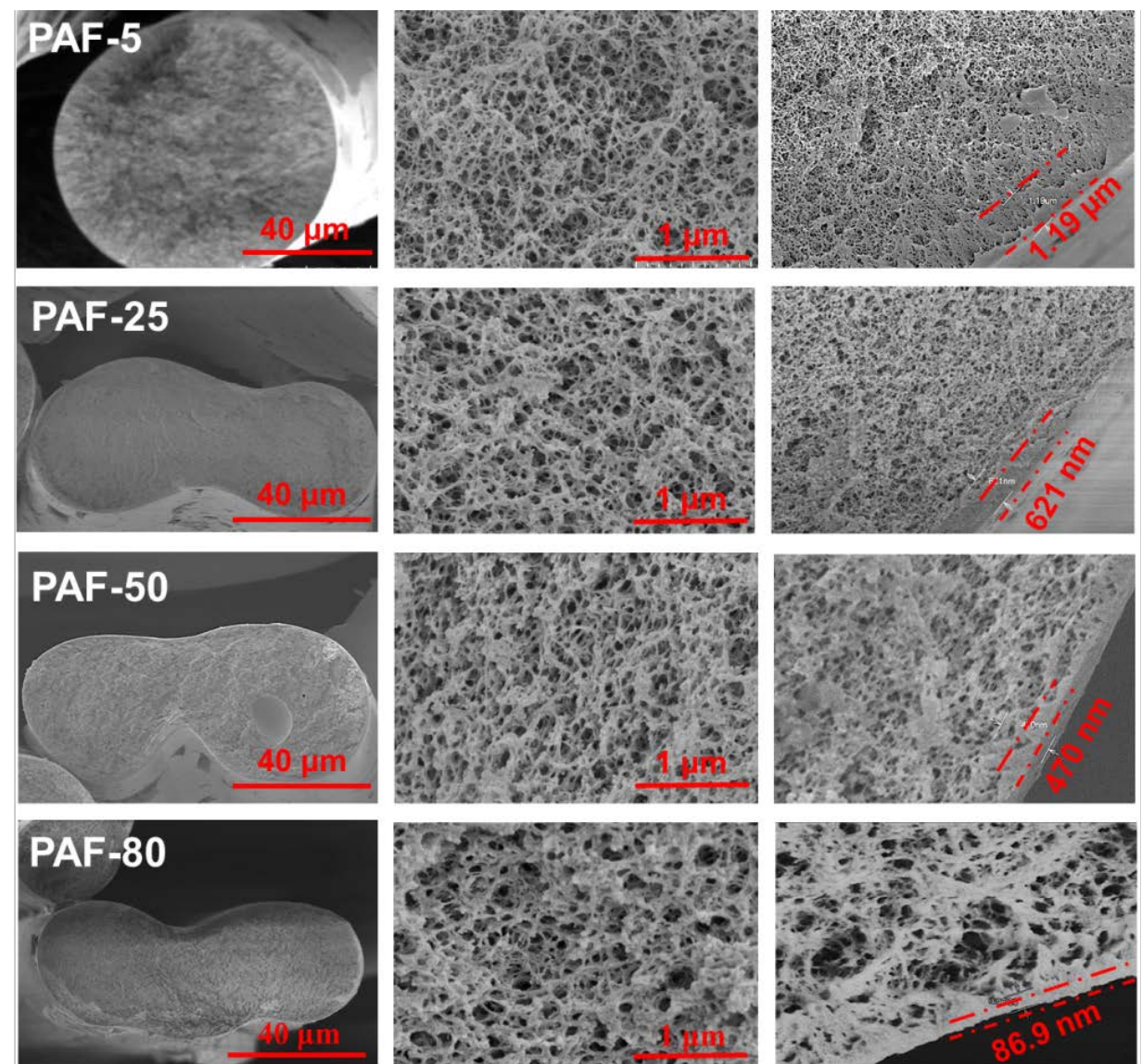

Figure S3. Cross-sectional SEM images with different magnifications of the PAFs prepared with the coagulation bath temperatures of $5,25,50$ and $80^{\circ} \mathrm{C}$, respectively. 


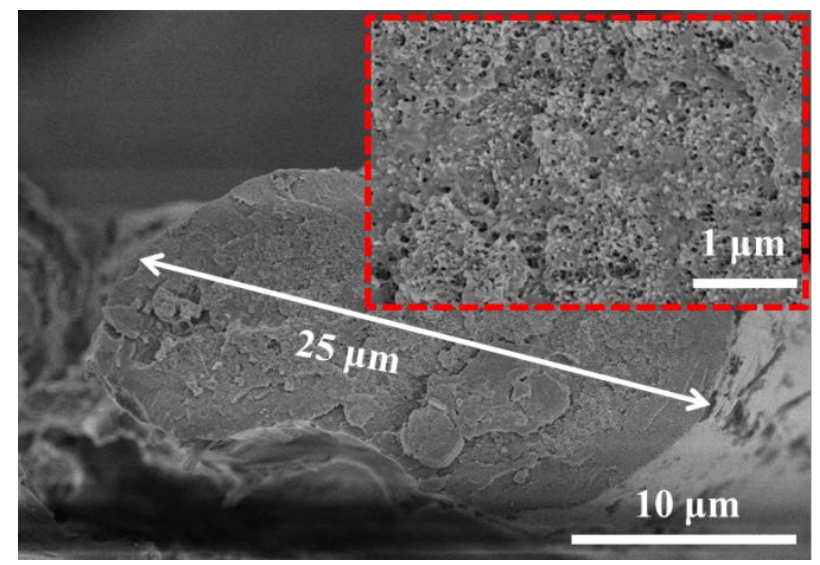

Figure S4. Cross-sectional SEM image of the PI fiber prepared via a conventional ambient pressure drying.
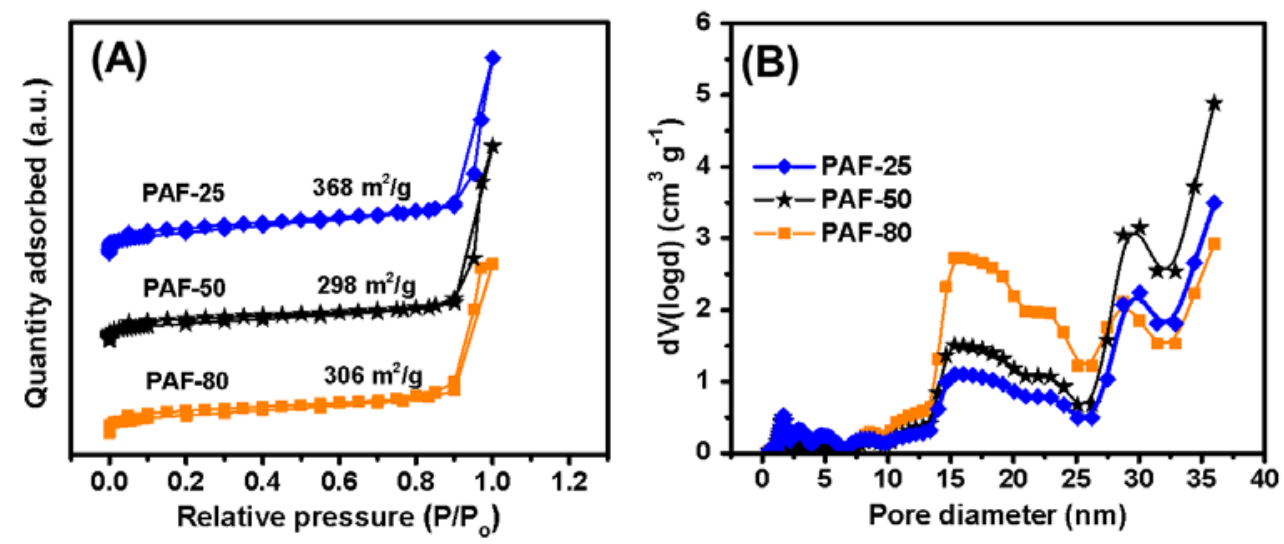

Figure S5. (A) Nitrogen adsorption/desorption isotherms of PAF-25, 50 and 80, and (B) pore-size distribution of PAFs calculated from the adsorption isotherms based on the density function theory (DFT) method. 

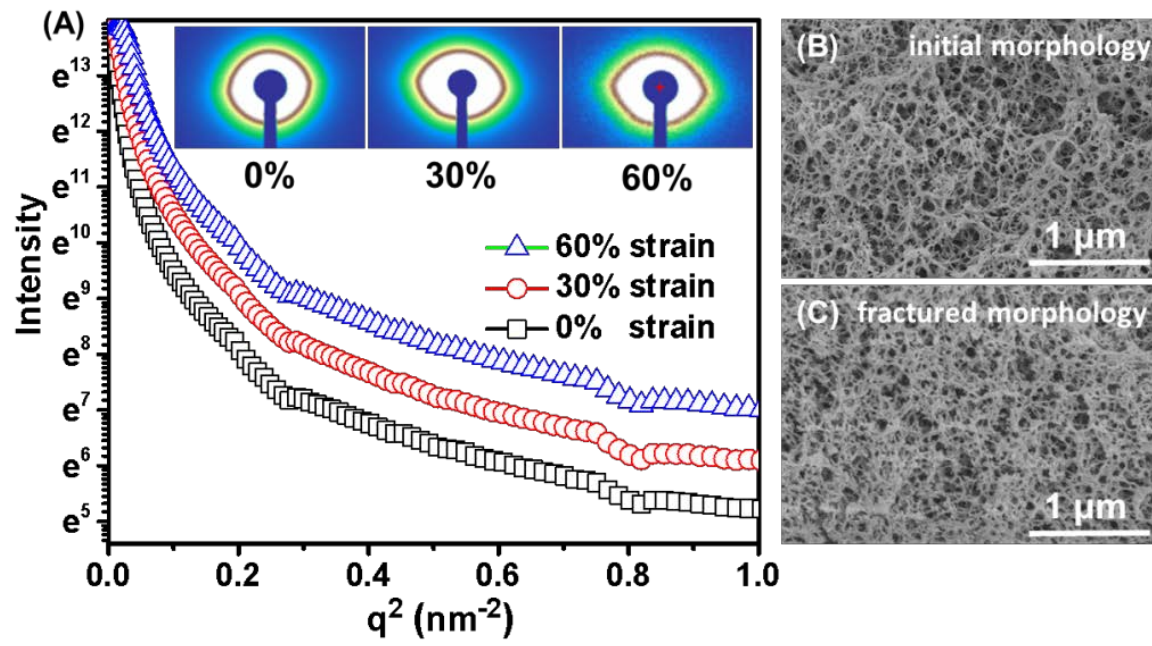

Figure S6. (A) Small-angle X-ray scattering (SAXS) of PAF measured at different strains. (B, C) Initial cross-section morphology and fractured morphology after tensile test of PAF-5.
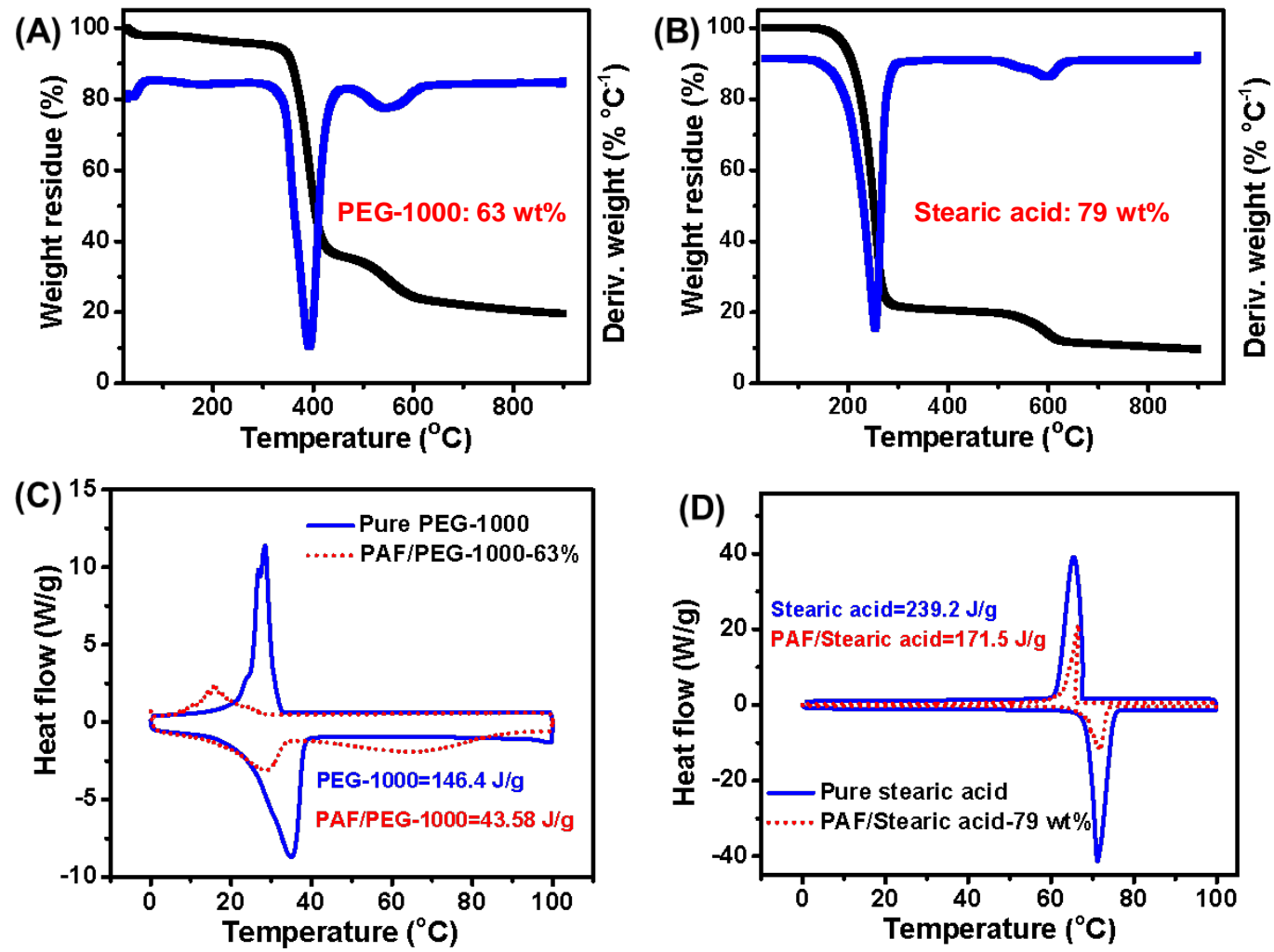

Figure S7. (A, B) TGA and DTG curves of PAF with PEG-1000 and stearic acid, respectively. (C) DSC curves of pure PEG-1000 and PAF/PEG-1000 composite fabric. (D) DSC curves of pure stearic acid and PAF/stearic acid composite fabric. 

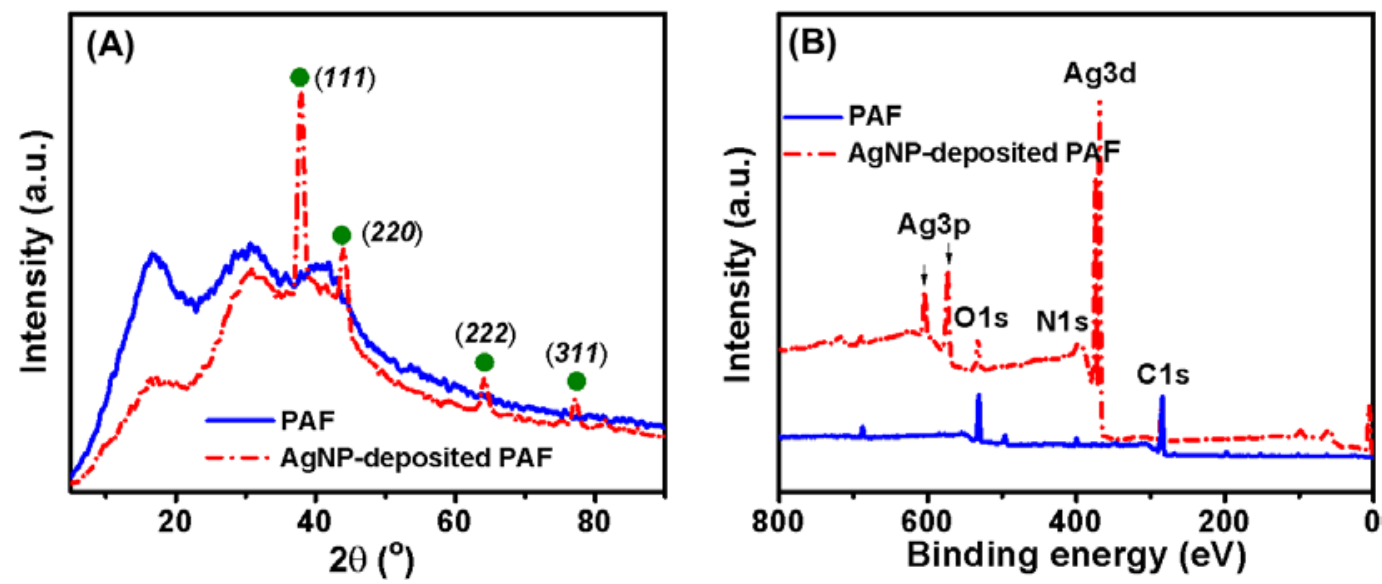

Figure S8. XRD (A) and XPS wide-scan spectra (B) of pristine PAF and Ag nanoparticle-deposited PAF (AgNP-deposited PAF) fibers.
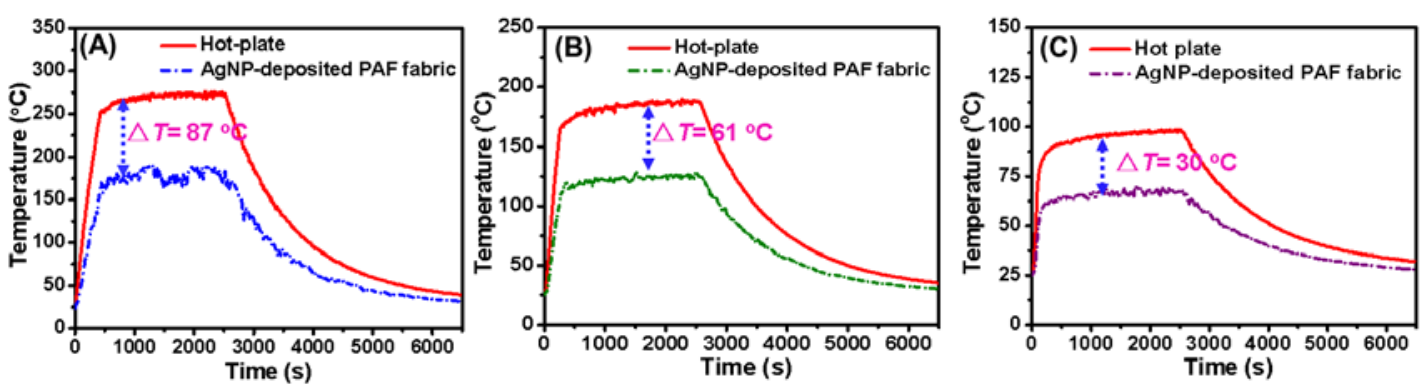

Figure S9. Temperature-time curves of the AgNP-deposited PAF fabrics at different temperatures: (A) $270{ }^{\circ} \mathrm{C}$, (B) $190{ }^{\circ} \mathrm{C}$, (C) $100{ }^{\circ} \mathrm{C}$.

Table S1. Solubility of synthesized polyimide in various solvents a

\begin{tabular}{ccccccccc}
\hline NMP & DMAc & DMF & DMSO & Acetone & THF & CHCl $_{3}$ & Methylbenzene & Xylene \\
\hline++ & ++ & ++ & ++ & ++ & +- & -- & -- & -- \\
\hline
\end{tabular}

${ }^{a}$ Qualitative solubility was measured with $1 \mathrm{mg}$ of a sample in $1 \mathrm{~mL}$ of organic solvent. ++ Soluble at room temperature; +- Partially soluble at room temperature; -- insoluble. 